\title{
Complex Regional Pain Syndrome Diagnosed with Triple-Phase Bone Scanning
}

Harleen Kaur, Mitchel Muhleman, and Helena R. Balon

Department of Diagnostic Radiology and Molecular Imaging, Beaumont Hospital, Royal Oak, Michigan

In this report, we present a case of complex regional pain syndrome in a 55-y-old woman in whom the diagnosis was made on the basis of the bone scan findings. We also discuss the typical and atypical scintigraphic presentations of this entity, including pathophysiology and management.

Key Words: complex regional pain syndrome; CRPS; bone scan

J Nucl Med Technol 2017; 45:243-244

DOI: $10.2967 /$ jnmt.117.192443

\section{$\mathbf{C}$}

omplex regional pain syndrome is a challenging clinical entity and requires a multidisciplinary approach. There is no single study or laboratory test that can confirm or exclude the diagnosis. In this report, we present a case of complex regional pain syndrome in a 55-y-old woman in whom the diagnosis was based on the bone scan findings. We also discuss the typical and atypical scintigraphic presentations of this entity, including pathophysiology and management.

\section{CASE REPORT}

A 55-y-old woman presented with pain and swelling of the right wrist 5 mo after undergoing open reduction and internal fixation for a right-wrist fracture. The patient was referred for a limited triple-phase bone scan for further evaluation. Immediately after injection of $851 \mathrm{MBq}$ of ${ }^{99 m}$ Tc-methylene diphosphonate, blood flow images (radionuclide angiogram) and blood pool images (soft-tissue phase) of the upper extremities were obtained, followed by delayed images at $3 \mathrm{~h}$ (Fig. 1).

Radiographs of the wrist demonstrated a fracture of the distal radius with Colles angulation (Fig. 2).

Bone scanning revealed the typical triple-phase positivity (increased blood flow activity, increased blood pool activity, and intense periarticular delayed uptake) in the small joints of the affected hand. The scintigraphic pattern

Received Feb. 27, 2017; revision accepted May 18, 2017.

For correspondence or reprints contact: Harleen Kaur, Beaumont Health, 3601 W. 13 Mile Rd., Royal Oak, MI 48073.

E-mail: harleen.kaur@beaumont.org

Published online Jun. 13, 2017.

COPYRIGHT (c) 2017 by the Society of Nuclear Medicine and Molecular Imaging. of periarticular accentuation in the osseous phase was characteristic of complex regional pain syndrome. The uptake in the right wrist was consistent with the recent fracture.

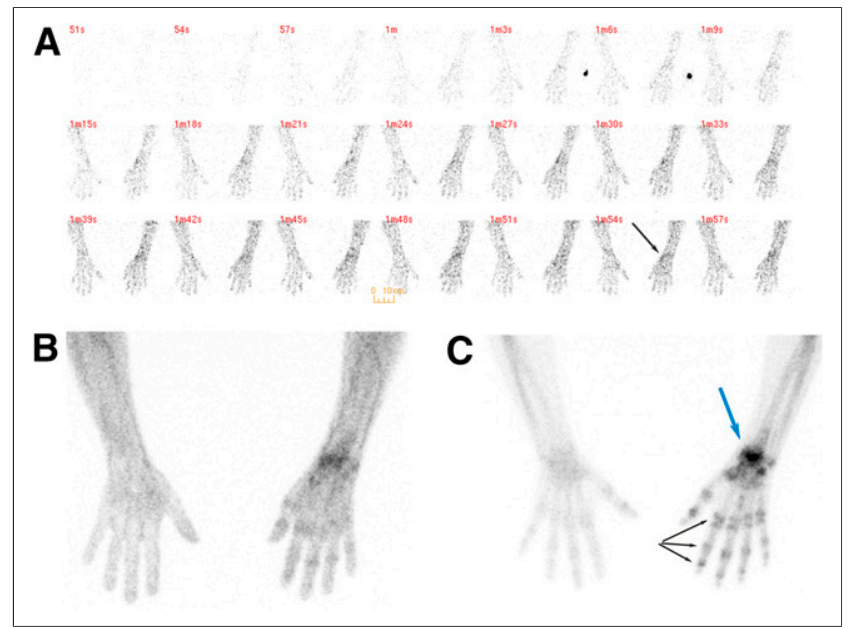

FIGURE 1. Typical presentation of complex regional pain syndrome. (A and B) Blood flow and blood pool images demonstrate increased activity diffusely in right hand and more focally in right wrist (arrow). (C) Delayed images demonstrate periarticular accentuation in multiple interphalangeal and metacarpophalangeal joints of involved hand (black arrows). Right wrist uptake (blue arrow) is consistent with recent fracture.

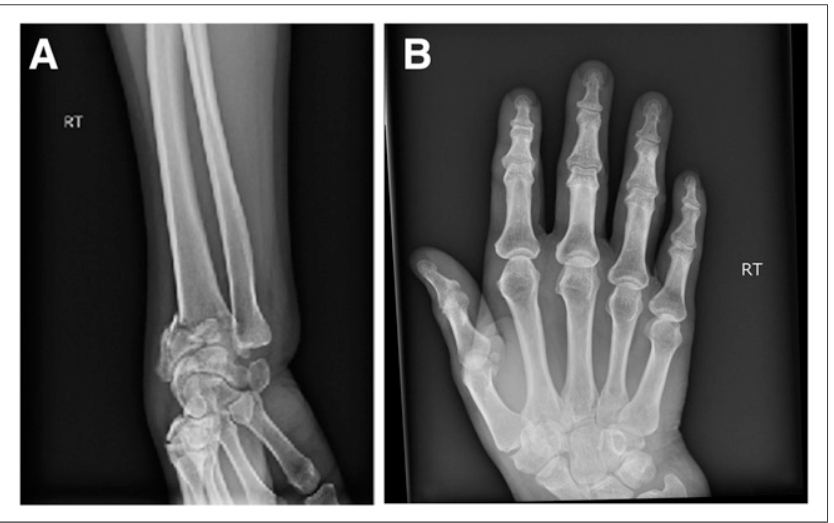

FIGURE 2. (A) Radiograph of wrist demonstrating fracture of distal radius with Colles angulation. (B) Radiograph of hand demonstrating no abnormality (B). 


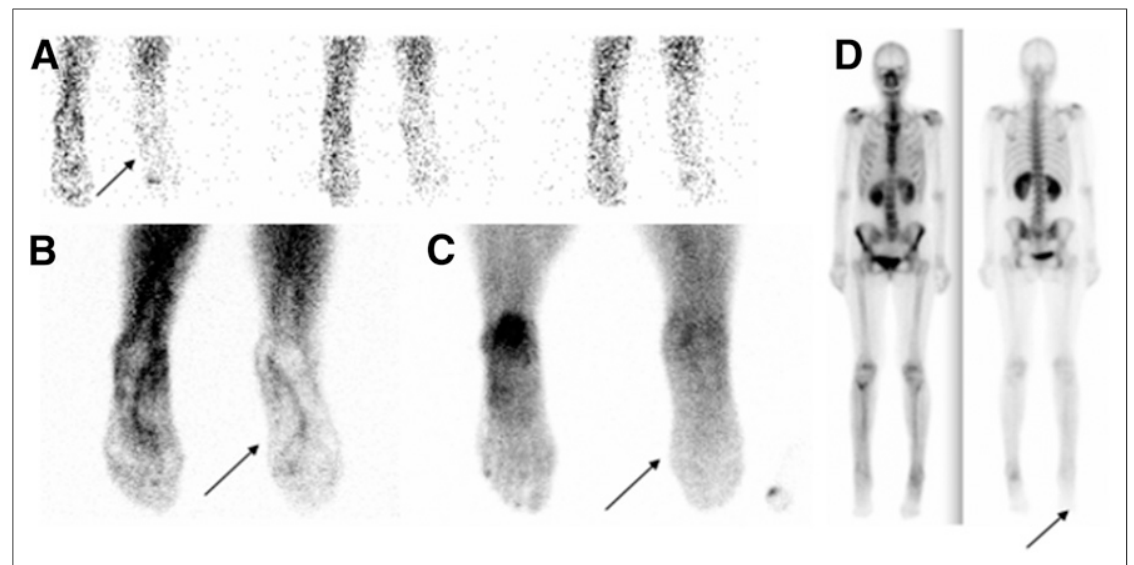

FIGURE 3. Example of atypical presentation of complex regional pain syndrome. A $45-y$-old woman presented with pain in right lower extremity for over $1 \mathrm{mo}$. Bone scanning demonstrated diminished radiotracer uptake (arrows) in right foot and distal leg in all 3 phases-blood flow (A), blood pool (B), and delayed (C)-as well as on whole-body images (D).

\section{DISCUSSION}

Our case demonstrates the typical scintigraphic pattern of complex regional pain syndrome. However, an atypical scintigraphic presentation may also be encountered, with decreased blood flow activity, decreased blood pool activity, and decreased radiotracer uptake in the bones and joints of the affected limb (Fig. 3). Such atypical presentations are usually seen in the late stages of the disease and in the pediatric population, in whom even the growth plates in the affected limb demonstrate decreased radiotracer accumulation.

The pathophysiology of complex regional pain syndrome remains unknown, but neurogenic inflammation is believed to play a crucial role in its development. The syndrome is associated with increased levels of proinflammatory cytokines (tumor necrosis factor $\alpha$, interleukin 6) and decreased levels of antiinflammatory cytokines (interleukin 1, interleukin 10 , tumor growth factor $\beta_{1}$ ) (1). Another possible mechanism is a vasomotor dysfunction accompanied by structural changes in the brain, which lead to sensitization (2).

The syndrome is managed with physical and occupational therapy, as well as with the following agents in early cases (2): nonsteroidal antiinflammatory drugs, anticonvulsant drugs (e.g., gabapentin or pregabalin), tricyclic or other antidepressant drugs (amitriptyline or nortriptyline), bisphosphonate drugs, and topical lidocaine or capsaicin cream. Refractory cases may require interventional procedures such as nerve blocks, sympathectomy, or neurostimulators.

Triple-phase bone scanning is one of the useful studies in this clinical situation, with a sensitivity of $87 \%$ according to a metaanalysis (3). Furthermore, triple-phase bone scanning enables exclusion of other diagnoses such as arthritis, pseudoarthropathy, benign or malignant bony lesions, and even metabolic bone diseases such as Paget disease, particularly if integrated SPECT/CT is added (4). The typical scintigraphic findings in complex regional pain syndrome are increased blood flow activity and increased blood pool activity due to hyperemia. Delayed images usually display the characteristic pattern of increased periarticular uptake in the small joints, as well as in the major joints of the affected limb, although other, less typical, patterns (decreased perfusion and delayed activity) are possible, especially in children and in the later stages of the disease.

\section{CONCLUSION}

Complex regional pain syndrome is a challenging clinical entity and requires a multidisciplinary approach. There is no single study or lab test that can confirm or exclude the diagnosis.

\section{DISCLOSURE}

No potential conflict of interest relevant to this article was reported.

\section{REFERENCES}

1. Pachowicz M, Nocuń A, Postępski J, Olesińska E, Emeryk A, Chrapko B. Complex regional pain syndrome type I with atypical scintigraphic pattern: diagnosis and evaluation of the entity with three phase bone scintigraphy - a case report. Nucl Med Rev Cent East Eur. 2014;17:115-119.

2. Freedman M, Greis AC, Marino L, et al. Complex regional pain syndrome: diagnosis and treatment. Phys Med Rehabil Clin N Am. 2014;25:291-303.

3. Ringer R, Wertli M, Bachmann LM, Buck FM, Brunner F. Concordance of qualitative bone scintigraphy results with presence of clinical complex regional pain syndrome 1: meta-analysis of test accuracy. Eur J Pain. 2012;16:1347-1356.

4. Usmani S, Panchadar S. Three-phase ${ }^{99 \mathrm{~m}}$ Tc-MDP bone scintigraphy and SPECT/ CT in pediatric pseudodystrophy. Clin Nucl Med. 2015;40:902-904. 